\title{
A Study on the Influence of Cyberbullying Characteristics on Attitudes toward Behaviors and Harmful Behaviors
}

\section{사이버불링 특성이 행위에 대한 태도와 가해행동에 미치는 영향에 관한 연구}

Chulgyu Park ${ }^{1}$, Hakyun Kim²

박철규 ${ }^{1}$, 김하균 ${ }^{2}$

${ }^{1}$ Docteral Candidate, 48513 Graduate School of Information System, Pukyong National Univ. 45, Yongso-ro, Nam-Gu. Busan, Korea, E-mail: tracck3651@naver.com

${ }^{2}$ Professor, 48513 Division of Business, Pukyong National Univ. 45, Yongso-ro, Nam-Gu. Busan, Korea, E-mail: kimhk@pknu.ac.kr

Corresponding author: Hakyun Kim

\begin{abstract}
Cyberbullying is emerging as a serious youth problem. In order to recognize and decrease the cyberbullying phenomenon from various fields, research from a realistic perspective has been continuously conducted. The scope of research on cyberbullying is also diverse, including definitions, characteristics, types, causes, and effects. In the case of Korea, as the information and communication industry develops, the age of use of information technology differs from elementary schoolchildren to the adult, so understanding of cyberbullying in cyberspace is of great importance. In order to intervene and prevent the perpetrators and sufferers in cyberbullying, it is essential to establish and empirically review cyberbullying research. This research emphases on investigating the connection between the characteristics of cyberbullying in cyberspace and the influence of the attitude toward behavior and the perpetrating behavior. As factors that determine the characteristics of cyberbullying, it was divided into anonymity, aggression, bystander, repeatability, and unrestriction. The results of the study is as follows. First, anonymity had a significant influence on attitudes toward behavior. Second, aggression had a significant effect on attitudes toward behavior. Third, bystander had a significant effect on attitudes toward behavior. Fourth, repetition had a significant effect on attitude toward behavior. Fifth, unrestriction had a significant effect on attitudes toward behavior. Sixth, the attitude toward behavior had a significant influence on the offending behavior.
\end{abstract}

Keywords: Anonymity, Aggression, Bystander, Repeatability, Unrestriction

요약: 사이버불링은 심각한 청소년 문제로 대두되고 있다. 사이버불링 현상을 다양한 방면에 서 이해하고, 줄이기 위한 현실적인 관점에서의 연구가 지속해서 이루어졌다. 사이버불링에 대한 연구범위도 정의, 원인, 결과, 특성, 유형, 등 범위가 넓게 연구되고 있다. 한국의 경우 정보통신산업이 발전함에 따라 정보기기의 사용연령도 초등학생에서부터 노년층까지 다양하 므로, 사이버공간에서 일어나는 사이버불링에 관한 이해는 중요성이 매우 크다. 사이버불링에 서 가해자와 피해자들에 대한 개입과 예방을 위해서는 사이버불링 연구에 대한 정리와 실증 
적 고찰이 필요하다. 본 연구는 사이버공간에서 사이버불링의 특성이 행위에 대한 태도와 가 해행동의 영향 관계를 분석하고 시사점을 제시하는데 초점을 둔다. 사이버불링의 특성을 결정 하는 요인으로 익명성, 공격성, 방관성, 반복성, 무제약성으로 나누었다. 연구의 결과는 다음과 같다. 첫째, 익명성은 행위에 대한 태도에 유의한 영향을 준다. 둘째, 공격성은 행위에 대한 태도에 유의한 영향을 준다. 셋째, 방관성은 행위에 대한 태도에 유의한 영향을 준다. 넷째, 반복성은 행위에 대한 태도에 유의한 영향을 준다. 다섯째, 무제약성은 행위에 대한 태도에 유의한 영향을 준다. 여섯째, 행위에 대한 태도는 가해행동에 유의한 영향을 준다.

핵심어: 익명성, 공격성, 방관성, 반복성, 무제약성

\section{1. 서론}

현재 사이버불링은 심각한 청소년 문제로 대두되고 있다. 사이버불링에 관한 연구는 2000년대를 시작으로 급속히 발전하여, 사이버불링으로 인한 자살, 학교폭력 등의 사이버 불링의 폐해가 보도됨에 따라 최근에 연구들이 활발해 지고 있다. 사이버불링 현상을 다 양한 방면에서 이해하고, 줄이기 위한 현실적인 관점에서의 연구가 지속해서 이루어졌다. 사이버불링에 대한 연구범위도 정의, 원인, 결과, 특성, 유형, 등 범위가 넓게 연구되고 있다. 한국의 경우 정보통신산업이 발전함에 따라 정보기기의 사용연령도 초등학생에서 부터 노년층까지 다양하므로, 사이버공간에서 발생하는 폭력 행위에 대한 이해는 중요성 이 매우 크다. 사이버불링에서 가해자와 피해자들에 대한 개입과 예방을 위해서는 사이 버불링 연구에 대한 정리와 실증적 고찰이 필요하다. 사이버불링에 의한 유형이나 특성 등의 연구는 많으나, 개인의 심리적인 영향과 관련된 연구는 미흡하여 객관적 데이터가 필요하다[1]. 최근에는 정보통신기기의 발달로 사이버불링의 피해가 증가하고 있어, 이와 관련된 연구는 중요하다.

블링(Bullying)이란 자신의 위치에서 직위를 이용해 상대방에게 신체적, 언어적, 사회적 피해를 주는 것이다. 이는 상대방을 위협하거나 당혹스럽게 만든다. 사이버불링은 상대방 에 대한 공격적 행위를 사이버공간에서 통신 기술 및 정보통신기기를 이용하여 고의적, 반복적, 지속해서 행하는 것이다[2]. 학교폭력에 대한 예방 차원에서 사이버불링은 태블 릿, 휴대전화, 컴퓨터 등 정보통신기기의 발달에 따라 학생들이 특정대상의 일부학생들을 지속적, 반복적으로 괴롭힘을 가하는 것이다. 또는 상대방에 대한 개인정보의 폭로 또는 허위사실을 사이트에 유포함으로 특정인을 괴롭히는 모든 행위이다.

본 연구는 행위에 대한 태도와 가해행위에 영향을 주는 요인인 사이버불링 특성을 익명성, 공격성, 방관성, 반복성, 무제약성으로 나누었다. 사이버 환경의 사이버불링 특성 이 행위에 대한 태도와 가해행동에 어떠한 영향을 미치는가를 실증적으로 분석하였다.

\section{2. 이론적 배경}

\section{1 사이버불링 특성}

\subsection{1 익명성}


사이버불링으로 상대방을 괴롭히기 쉬운 환경이라 할 수 있으며, 사이버불링이 쉽다고 인식하는 주된 이유로 익명성이다[3]. 사이버공간에서는 익명성의 보장은 개인의 신분이 드러나지는 않으며, 처벌에 대하여 걱정할 필요가 없고, 피해자의 반응에 신경 쓸 필요도 없으며, 죄책감을 느끼지도 않는다[4]. 오프라인상에서는 타인이나 가족의 시선을 의식하 여 공격적 행위를 나타내지 못했던 사람도 온라인상에서는 익명으로 타인에게 피해를 줄 수 있다. 사이버불링 가해자의 대부분이 피해자와 관련이 있지만, 소수의 피해자만이 가 해자를 안다는 연구결과가 있다[5]. 따라서 사이버불링 피해의 대부분이 익명으로 행해지 고 있다. 익명성은 사이버공간이라는 특성을 이용하여 오프라인에서는 가능하지 않는 행 동을 한다. 사이버불링은 전통적 블링과 중복하여 발생하는 경우가 많다.

\subsection{2 공격성}

사이버불링 가해자를 상담할 때 가장 중요하게 다뤄야 하는 심리적 요인은 공격성이다. 청소년들이 자신의 공격에너지의 창구로 사이버불링을 사용한다[6]. 공격적 환상을 드러 내는 통로로 사이버불링을 찾는다고 보아야 할 것이다. 공격성이 높을수록 사이버불링 가해경험이 높다. 악성댓글을 만드는 사람은 상대방을 가해할 목적이 있으며, 상대방을 가해후에도 죄책감을 느끼지 않는다. 언어적 폭력 성향이 강할수록, 자기통제력이 약할수 록 사이버공간에서 행동을 많다고 밝혔다. 공격성에 관한 연구들을 종합하면, 오프라인에 서 상대방을 가해한 청소년들이 온라인에서도 공격적이며, 공격성을 평상시에 숨기고 있 던 사람이 공격성을 표출하는 통로로 사이버공간을 사용할 수도 있다.

\subsection{3 방관성}

일반적으로 전통적인 집단 괴롭힘에 대해 무관심하거나 방관의 태도를 나타내고 있다. 집단 괴롭힘의 기간이 오래될수록 방관자가 늘어나면서 집단 괴롭힘에 가세한다. 사이버 불링의 경우에는 익명성으로 인한 방관자의 수가 매우 많으므로 책임자가 분산되며, 전 통형 집단 괴롭힘보다 중재자의 역할이 어렵다. 방관자는 전통적 괴롭힘보다 사이버공간 에서 중요한 역할을 한다. 온라인상에서 괴롭힘이 발생했을 때 참여자들이 실제 사이버 불링을 저지하기 위해 의지가 있는 참여자들은 거의 없다[7]. 방관자가 사이버불링에 막 대한 영향을 미치고 있음에도 불구하고, 방관자 대부분은 사이버불링을 목격했을 때 수 동적으로 된다.

\subsection{4 반복성}

사이버불링이 고통스럽게 느껴지는 것은 계속되는 반복과 지속 때문이다. 오프라인 폭 력이 온라인으로 변화되면서 협박이나 괴롭힘이 오랜 기간 반복, 지속한다[8]. 사이버공간 의 특성상 여러 명이 반복하게 되므로 피해자는 지속적인 사이버불링을 피할 수 없다. 행위의 반복성을 공격 행동을 구분하는 중요한 기준으로 보고 있다[9]. 사이버공간에서의 괴롭힘의 반복성은 현실 공간에서 이루어지는 괴롭힘 행위와 확실히 다르다. 인터넷 공 간에서 사이버불링은 가해자의 통제로부터 무방비하게 다양한 형태로 반복된다. 게재된 댓글, 메일이 다른 사람에게 접속하여 열람 또는 전송된다. 특히, 대중적인 관심이 있는 상황의 경우는 기하급수적으로 늘어난다. 일반적인 집단 괴롭힘에서는 가해자 본인이 지 속적인 반복성이 특징으로서 강조되었으나, 사이버불링의 경우에는 가해자와 다른 사람 
의 방관도 반복성에 가담한다고 본다.

\subsection{5 무제약성}

사이버공간에서는 공간과 시간의 제약이 없다. 어떤 사람이든지 마음만 먹으면 사이버 공간에서 24시간 동안 사용할 수 있으며, 다양한 장소에서 어려움 없이 인터넷 사이트에 접속할 수 있다[9]. 사이버불링에 참여한 사람들은 시간과 공간에 제약이 없다. 따라서 사 이버불링 피해자를 목표로 삼아 계속적, 지속해서 사진, 비디오, 및 소셜미디어, 채팅 등 의 다양한 미디어를 사용한다. 일회성 사이버불링도 시간과 공간의 제약이 없으므로, 다 양한 방법으로 피해자를 괴롭힐 수 있다. 상대방에게 새로운 형태로 반복해서 괴롭힘을 줄 수 있다. 사이버불링 피해자의 심리적, 정서적 고통은 지속적, 반복적으로 가중 된다. 시간적, 공간적으로 무 제약성은 광범위하고 국가를 초월한 형태로 발전하고 있다.

\section{2 행위에 대한 태도}

사이버불링의 심리적 측면을 연구하는 과정에서 개인적인 내부 심리에 관한 변수들을 합리적 행동이론에서 가져오고 있다[10]. 여러 외생 변수 중에서 주관적 규범과 개인의 행위에 대한 태도가 수용의도에 영향을 미친다는 것이다. 일반적으로 행동예측에 대한 대표적인 이론으로는 합리적 행위이론을 들 수 있다. 이는 행위에 대한 태도와 주관적 규범이 행동의도에 영향을 준다고 보았다. 계획된 행동이론의 행동의도를 결정하는 결정 요인 중에서 첫 번째 예측요인이 행동에 대한 태도이다. 행동에 대한 개인이 가진 긍정 이나 부정의 평가 정도를 말한다. 계획된 행동이론에서 행위에 대한 태도는 심리적인 개 인의 특정행동에 대한 신념(belief)의 측정과 평가를 의미한다[11]. 행위에 대한 태도는 행 동에 대한 종합적인 느낌의 평가이다. 즉, 행위에 대한 태도는 특정행위에 대한 총체적인 심리적, 정서적 평가를 의미한다.

\section{3 가해행동}

가해자의 특성을 살펴보면, 사이버 피해를 주는 것은 오락적인 가치가 있다는 것을 인지함으로써 단순한 재미로 하기도 한다. 단순히 글을 옮기거나 링크함으로 가해자의 의도와 상관없이 또는 모르게 가해하는 특성이 있다. 결과적으로 자신의 행동이 자신도 모르게 사이버불링이 결행 될 수 있다. 사이버불링 가해행동 관련 사회, 환경적 요인들의 경우, 학교폭력을 포함한 학교생활에서의 적응이 중요한 변수로 드러났다. 연구들이 사이버불링을 동료 간의 괴롭힘의 하위 유형에 초점을 맞추고 있다[12]. 피해경험과 가해행동은 중복되는 경우가 빈번하며, 피해경험이 가해행동을 예측하는 것으로 나타났다[13].

\section{3. 연구설계}

\section{1 연구모형}

본 연구는 사이버 환경에서 사이버불링 특성이 행위에 대한 태도와 가해행동의 영향 
관계를 분석하는 데 초점을 둔다. 사이버불링 특성을 결정하는 요인으로 익명성, 공격성, 방관성, 반복성 및 무제약성으로 나누었다[3-9]. 행위에 대한 태도와 가해행동은 선행연구를 바탕으로 연구모형을 제시하면 [그림 1]과 같다[10-13].

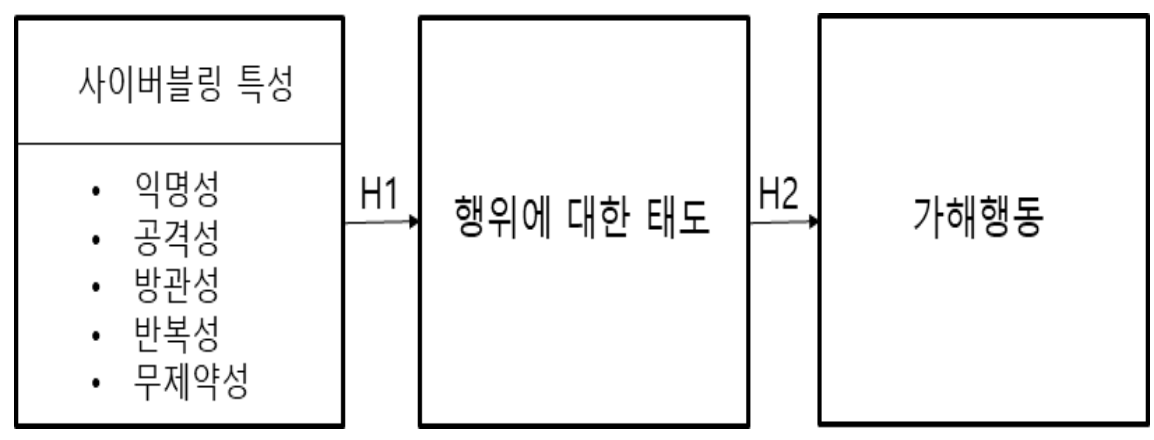

[그림 1] 연구모형

[Fig. 1] Research Model

\section{2 연구가설}

사이버불링 가해자를 상담할 때 가장 중요하게 다뤄야 하는 심리적 요인은 익명성, 공 격성이다. 청소년들이 자신의 공격에너지의 창구로 사이버불링을 사용한다[7]. 따라서 방 관자의 행동에 따라 사이버불링을 변화시키거나 영향력을 미칠 수 있다. 방관자는 피해 자를 지지하며 개입할 수 있다. 사이버불링이 고통스럽게 느껴지는 것은 계속되는 반복 과 지속 때문이다. 오프라인 폭력이 온라인으로 변화되면서 협박이나 괴롭힘이 오랜 기 간 반복, 지속한다[8]. 본 연구에서는 선행연구를 기반으로 다음과 같은 가설을 설정하였 다.

가설 H1-1 익명성은 행위에 대한 태도에 따라 차이가 있을 것이다.

가설 H1-2 공격성은 행위에 대한 태도에 따라 차이가 있을 것이다.

가설 H1-3 방관성은 행위에 대한 태도에 따라 차이가 있을 것이다.

가설 H1-4 반복성은 행위에 대한 태도에 따라 차이가 있을 것이다.

가설 H1-5 무제약성은 행위에 대한 태도에 따라 차이가 있을 것이다.

계획된 행동이론에서 행위에 대한 태도는 심리적인 개인의 특정행동에 대한 신념 (belief)의 측정과 평가를 의미한다[11]. 연구들이 사이버불링을 동료 간의 괴롭힘의 하위 유형에 초점을 맞추고 있다[12]. 본 연구에서는 선행연구를 기반으로 다음과 같은 가설을 설정하였다.

가설 $\mathrm{H} 2$ 행위에 대한 태도는 가해행동에 따라 차이가 있을 것이다.

\section{4. 실중분석 및 결과}

설문지의 기본통계분석을 위해 통계패키지인 SPSS 22.0 을 사용하였다. 가설검정을 위해 
구조방정식 패키지 Smart PLS 2.0을 사용하여 집중타당성과 판별타당성을 검증하였다.

\section{1 자료수집 및 표본의 특성}

총 210 명의 사용자가 설문조사에 응답했다. 불성실한 응답 7 부를 제외하고 모두 203부가 기본적인 통계조사에 사용되었다. 응답자의 $43 \%$ 는 중고등학교 학생이고, $57 \%$ 는 대학생과 일반인이다. 일반인이 학생보다 높은 이유는 대학생이 포함되어 있기 때문이다. 평균연령은 23.4 세였다. 설문조사응답자는 대부분이 학생과 일반인이었으며, 전체연령대는 낮았다. 연령별 분포를 살펴보면 일반인이 $13 \%$ 와 대학생이 $44 \%$, 중학교 $23.6 \%$, 고등학교 $19.4 \%$ 였다. 일반인의 경우 40 대 이상은 $3.2 \%$ 로 참여도가 낮았다. 사이버불링의 표본은 많은 연구가 중학교, 고등학교 위주로 되어있다. 하지만 계속적으로 사이버불링의 연령이 증가하는 추세에 있습니다. 40 대 이상의 경우 아직은 사이버불링의 경우가 적다고 할 수 있습니다.

연구대상자의 인구사회학적 특성은 기존의 많은 연구가 진행되어 온 것처럼 중고등학생이 $50 \%$ 를 차지하였으며, 본 연구에서 사이버불링의 피해자나 가해자가 대학생의 경우 $44 \%$ 를 이루었다는 것이 또 다른 특성이다.

\section{2 자료의 분석방법}

집중 타당성의 결과는 [표 1]과 같다. 판별타당성의 결과는 [표 2]와 같다.

[표 1] 신뢰도 및 집중 타당성 분석결과

[Table 1] Reliability and Internal Consistency Results

\begin{tabular}{|c|c|c|c|c|}
\hline 변수 & 요인적재 값 & AVE & C. R. & Cronbach's \\
\hline \multirow{4}{*}{ 익명성 } & 0.831 & \multirow{4}{*}{0.634} & \multirow{4}{*}{0.896} & \multirow{4}{*}{0.877} \\
\hline & 0.754 & & & \\
\hline & 0.783 & & & \\
\hline & 0.822 & & & \\
\hline \multirow{3}{*}{ 공격성 } & 0.923 & \multirow{3}{*}{0.862} & \multirow{3}{*}{0.949} & \multirow{3}{*}{0.920} \\
\hline & 0.938 & & & \\
\hline & 0.923 & & & \\
\hline \multirow{3}{*}{ 방관성 } & 0.784 & \multirow{3}{*}{0.675} & \multirow{3}{*}{0.892} & \multirow{3}{*}{0.856} \\
\hline & 0.865 & & & \\
\hline & 0.827 & & & \\
\hline \multirow{3}{*}{ 반복성 } & 0.859 & \multirow{3}{*}{0.734} & \multirow{3}{*}{0.892} & \multirow{3}{*}{0.820} \\
\hline & 0.882 & & & \\
\hline & 0.828 & & & \\
\hline \multirow{3}{*}{ 무제약성 } & 0.651 & \multirow{3}{*}{0.510} & \multirow{3}{*}{0.804} & \multirow{3}{*}{0.801} \\
\hline & 0.688 & & & \\
\hline & 0.643 & & & \\
\hline \multirow{2}{*}{ 행위에 대한 태도 } & 0.958 & \multirow{2}{*}{0.872} & \multirow{2}{*}{0.953} & \multirow{2}{*}{0.926} \\
\hline & 0.936 & & & \\
\hline
\end{tabular}




\begin{tabular}{|c|c|c|c|c|}
\hline & 0.906 & & & \\
\hline \multirow{4}{*}{ 가해행동 } & 0.935 & \multirow{4}{*}{0.881} & \multirow{4}{*}{0.967} & \multirow{4}{*}{0.955} \\
\hline & 0.942 & & & \\
\hline & 0.947 & & & \\
\hline & 0.930 & & & \\
\hline
\end{tabular}

[표 2] 상관관계 및 판별 타당성 분석결과

[Table 2] Correlation and Discriminant Validity

\begin{tabular}{|c|c|c|c|c|c|c|c|c|}
\hline 변수 & AVE & 1 & 2 & 3 & 4 & 5 & 6 & 7 \\
\hline 익명성 & 0.634 & $\mathbf{0 . 7 9 6}$ & & & & & & \\
\hline 공격성 & 0.862 & 0.629 & $\mathbf{0 . 9 2 8}$ & & & & & \\
\hline 방관성 & 0.675 & 0.723 & 0.609 & $\mathbf{0 . 8 2 1}$ & & & & \\
\hline 반복성 & 0.734 & 0.360 & 0.172 & 0.309 & $\mathbf{0 . 8 5 6}$ & & & \\
\hline 무제약성 & 0.510 & 0.694 & 0.510 & 0.663 & 0.356 & $\mathbf{0 . 7 1 4}$ & & \\
\hline 행위에 대한 태도 & 0.872 & 0.758 & 0.452 & 0.796 & 0.248 & 0.696 & $\mathbf{0 . 9 3 3}$ & \\
\hline 가해행동 & 0.881 & 0.578 & 0.476 & 0.437 & 0.355 & 0.445 & 0.386 & $\mathbf{0 . 9 3 6}$ \\
\hline
\end{tabular}

\section{3 연구 모형의 검증}

구조모형은 Smart PLS 2.0을 사용하였으며 구조모형을 통해서는 연구모형의 변수들 간 의 경로계수와 결정계수(R2)결과 값을 도출하였다.

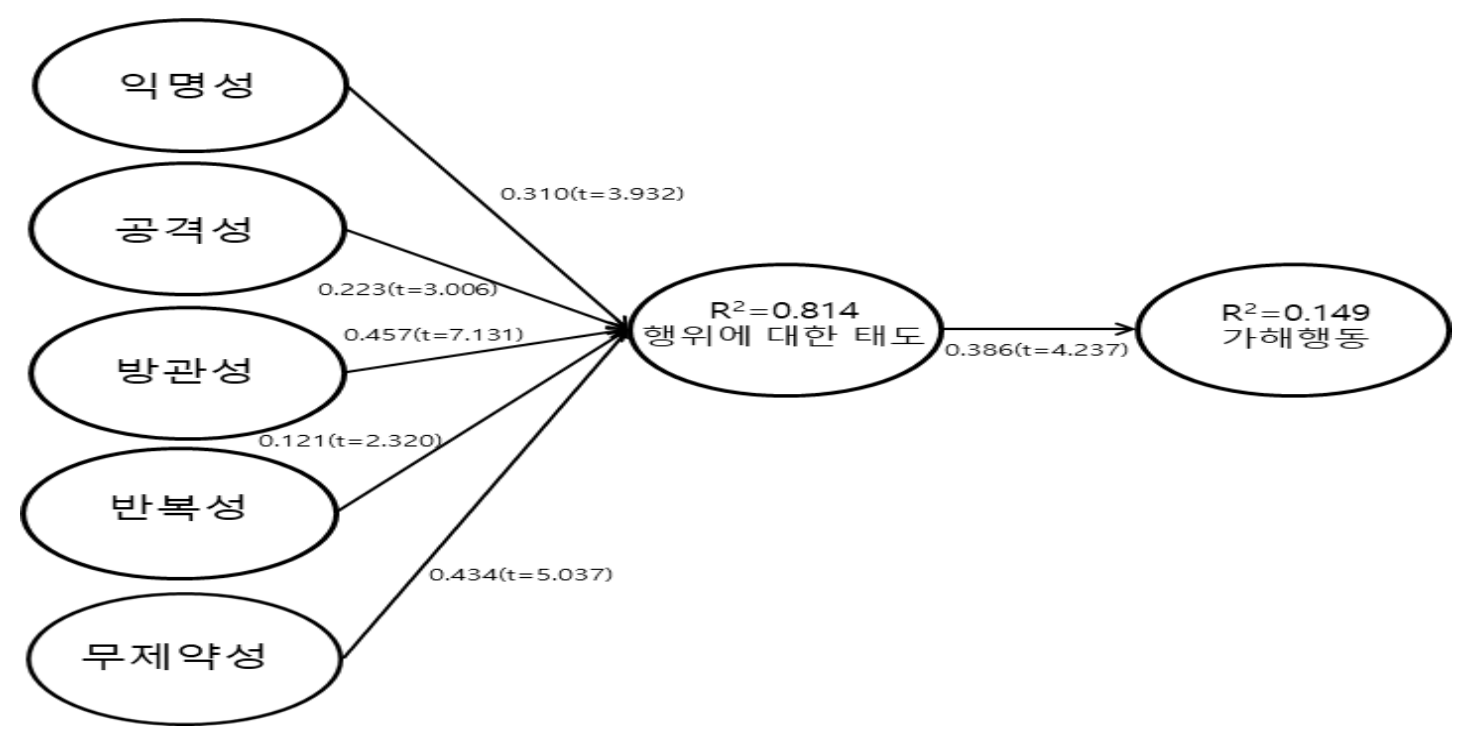

[그림 2] 모형 분석결과

[Fig. 2] The Results of Research Model 
제안한 가설 $\mathrm{H} 1, \mathrm{H} 2$ 는 채택되었다. 첫째, 가설 $\mathrm{H} 1-1$ 의 "익명성은 행위에 대한 태도에 따라 차이가 있을 것이다"라는 가설은 채택되었다. 익명성과 행위에 대한 태도 $(\beta=0.310$, $\mathrm{t}=3.932, \mathrm{p}<0.05)$ 는 유의한 영향을 미치는 것으로 나타났다. 이는 사이버불링에서 익명성은 행위에 대한 태도에 영향을 준다는 것이다. 이는 기존의 연구[3][4]와 같다. 둘째, 가설 H1-2의 "공격성은 행위 대한 태도에 따라 차이가 있을 것이다"라는 가설은 채택되었다. 공격성과 행위에 대한 태도 $(\beta=0.223, \mathrm{t}=3.006, \mathrm{p}<0.05)$ 는 유의한 영향이 있는 것으로 나타났다. 이는 사이버불링에서 공격성은 행위에 대한태도에 영향을 준다는 것이다. 이는 기존의 연구[7]와 같다. 셋째, 가설 H1-3의 "방관성은 행위에 대한 태도에 따라 차이가 있을 것이다"라는 가설은 채택되었다. 방관성과 행위에 대한 태도( $\beta=0.457, \mathrm{t}=7.131$, $\mathrm{p}<0.05)$ 는 유의한 영향을 미치는 것으로 나타났다. 이는 기존의 연구[1]와 같다. 넷째, 가설 H1-4의 "반복성은 행위에 대한 태도에 따라 차이가 있을 것이다"라는 가설은 채택되었다. 반복성과 행위에 대한 태도 $(\beta=0.121, \mathrm{t}=2.320, \mathrm{p}<0.05)$ 는 유의한 영향을 미치는 것으로 나타났다. 이는 기존의 연구[8][9]와 동일하다. 다섯째, 가설 H1-5의 "무제약성은 행위에 대한 태도에 따라 차이가 있을 것이다"라는 가설은 채택되었다. 무제약성과 행위에 대한 태도 $(\beta=0.434, \mathrm{t}=5.037, \mathrm{p}<0.05)$ 는 유의한 영향을 미치는 것으로 나타났다. 이는 기존의 연구[10]과 같다. 여섯째, 가설 $\mathrm{H} 2$ 의 "행위에 대한 태도는 가해행동에 따라 차이가 있을 것이다"라는 가설은 채택되었다. 행위에 대한 태도와 가해행동 $(\beta=0.386$, $\mathrm{t}=4.237, \mathrm{p}<0.05)$ 은 유의한 영향을 미치는 것으로 나타났다. 이는 기존의 연구[10-12]와 같다.

[표 3] 가설검정 결과 요약

[Table 3] Results of Hypothesis Tests for the Research Model

\begin{tabular}{|c|c|c|c|}
\hline 가설 경로 & 표준화 계수 $(\beta)$ & t-value & 채택 유무 \\
\hline H1.1: 익명성 $\rightarrow$ 행위에 대한 태도 & 0.310 & 3.932 & 채택 \\
\hline H1.2: 공격성 $\rightarrow$ 행위에 대한 태도 & 0.223 & 3.006 & 채택 \\
\hline H1.3: 방관성 $\rightarrow$ 행위에 대한 태도 & 0.457 & 7.131 & 채택 \\
\hline H1.4: 반복성 $\rightarrow$ 행위에 대한 태도 & 0.121 & 2.320 & 채택 \\
\hline H1.5: 무제약성 $\rightarrow$ 행위에 대한 태도 & 0.434 & 5.037 & 채택 \\
\hline H2: 행위에 대한 태도 $\rightarrow$ 가해행동 & 0.386 & 4.237 & 채택 \\
\hline \multicolumn{4}{|c|}{$\mathrm{t}=1.97^{*}, \mathrm{p}<0.05$} \\
\hline
\end{tabular}

\section{5. 결론}

본 연구에서는 사이버불링 특성(익명성, 공격성, 방관성, 반복성, 무제약성)이 행위에 대 한 태도를 매개로 가해행동과 의 관계를 알아보기 위하여 실증적으로 연구하였다. 사이 버불링 특성, 행위에 대한 태도 및 가해행동에 대한 기존 문헌을 정리하였다. 기존연구를 바탕으로 사이버불링 특성, 행위에 대한 태도, 가해행동에 대한 연구모형과 연구가설을 
설정하였다. 본 연구를 통하여 사이버불링 특성이 행위에 대한 태도와 가해행동에서 규 명된 것은 다음과 같다. 사이버불링 특성이 행위에 대한 태도와 가해행동에 유의하다는 것이 실증적으로 확인되었다. 사이버불링 특성이 가해행동의 예측에 유용한 이론이라는 것을 다시 확인하였다. 따라서 다음과 같은 시사점을 제시할 수 있다. 첫째, 사이버불링 의 특성에 관한 연구이다. 사이버불링의 다양한 연구가 진행되고 있지만, 사이버불링을 해결하기 위해서는 개인적이고 심리적인 측면을 고려한 사이버불링의 세심한 연구가 필 요하다. 특히 문제가 되는 중고등학교의 사이버불링은 사이버 환경을 고려하여 교육기간 의 차별화된 사이버불링의 교육이 필요하다. 사이버불링을 해결하려는 교육기관은 인지 적 공감능력, 사이버 윤리의식 교육 및 함양을 위한 프로그램 실행이 필요하다. 이러한 해결방법의 다양한 조화를 통해서 사이버폭력의 해결이 가능하다. 둘째, 사이버불링 특성 (익명성, 공격성, 방관성, 반복성, 무제약성)은 행위에 대한 태도에 영향을 미치는 것으로 나타났다. 향후 발전적으로 사이버불링을 없애기 위해서는 이 외에도 다양한 사이버불링 특성이 개발되어야 할 것이다. 사이버불링에서 가해행동을 유발하는 정서가 중요한 연구 주제이므로 향후 세부적으로 진행되어야 한다. 현재 연구들은 사이버불링의 가해자들에 관한 연구라고 볼 수 있다. 사이버불링의 피해자가 가해행동을 하는 사례가 많으므로, 피 해자와 가해자를 동시에 연구해야 할 필요가 있다. 자기 통제력이 낮은 청소년이 사이버 불링 가해행동을 유발할 수 있다는 것이다. 사이버불링이라는 문제의 해결을 위해 사이 버불링 특성에 대한 세부적 요인들을 살펴보고 가해행동에 대한 문제를 해결할 수 있는 연구가 진행되어야 할 것이다.

\section{References}

[1] Jieun Lee, S. C. Jong, Ontology Development of Adolescent Cyberbullying, (2021), Vol.28, No.4, pp.163-192, DOI : 10.21509/KJYS.2021.04.28.4.163.

[2] J. Barliskal, A. Szuster, M. Winiewski, Cyberbullying among Adolescent Bystanders: Role of the Communication Medium, form of Violence, and Empathy, Journal of Community \& Applied Social Psychology, (2013), Vol.23, No.1, pp.37-51, https://doi.org/10.1002/casp.2137

[3] D. M. Law, J. D. Shapka, S. Hymel, B. F. Olson, T. Waterhouse, The Changing Face of Bullying: An Empirical Comparison between Traditional and Internet Bullying and Victimization, Computers in Human Behavior, (2012), Vol.28, No.1, pp.226-232, https://doi.org/10.1016/j.chb.2011.09.004

[4] Mckenna, K. Y. A, Influence on the Nature and Functioning of Social Groups, Psychological Aspects of Cyberspace: Theory, Research and Applications, Cambridge University Press, (2008), pp.228-242, https://doi.org/10.1017/CBO9780511813740.011

[5] J. You, H. Hong, The Relationship between Self-Efficacy and Bystander Defending Behavior in Cyberbullying among University Students: The Mediating Effects of Moral Disengagement and Guilt, Korean Journal of Counseling and Psychotherapy, (2020), Vol.32, No.4, pp.1647-1668, DOI : 10.23844/kjcp.2020.11.32.4.1647

[6] R. M. Kowalski, S. P. Limber, P. W. Agatston, Cyberbullying: Bullying in the Digital Age, (2nd Edition), WileyBlackwel, (2012)

[7] K. Chu, J. Choi, S. Lee, A Study on Factors Influencing on Flaming Behavior in Cyberspace, Journal of Cultural Industry Studies, (2013), Vol.13, No.2, pp.47-57, UCI : G704-SER000010126.2013.13.2.011

[8] J. W. Patchin, S. Hinduja, Bullies Move Beyond the Schoolyard: A Preliminary Look at Cyberbullying, Youth Violence and Juvenile Justice, (2006), Vol.4, No.2, pp.148-169, https://doi.org/10.1177/1541204006286288 
[9] D. Olweus, Bullying at School: What We Know and What We Can Do Wiley-Blackwell, (1993)

[10] W. So, H. Kim, A Study on the Determinants of Behavioral Theory Planned in Cyberbling, Asia-pacific Journal of Multimedia Services Convergent with Art, Huminites, and Sociology, (2019), Vol.9, No.10, pp.597-606, DOI : 10.35873/ajmahs.2019.9.10.051

[11] H. Vandebosch, K. V. Clemput, Cyberbullying Among Youngsters: Profiles of Bullies and Victims, New Media \& Society, (2009), Vol.11, No.8, pp.1349-1371, https://doi.org/10.1177/1461444809341263

[12] K. A. Fanti, A. G. Demetriou, V. V. Hawa, A longitudinal study of cyberbullying: Examining risk and protective factors, European Journal of Developmental Psychology, (2012), Vol.9, No.2, pp.168-181, https://doi.org/10.1080/17405629.2011.643169

[13] D. S. Wong, H. C. Chan, C. H. Cheng, Cyberbullying Perpetration and Victimization among Adolescents in Hong Kong, Children and Youth Services Review, (2014), Vol.36, pp.13-140, https://doi.org/10.1016/j.childyouth.2013.11.006 